\section{Pesticide resistance on the increase, says UNEP}

RESISTANCE to pesticides has been spreading so rapidly among pests that no manufacturer cared to offer a new pesticide to the World Health Organisation for safety testing in 1978. It now takes millions of dollars to test the safety of new chemicals and often within months pests start developing resistance to it. WHO recently reported that it is now cutting down its field staff involved in safety testing of pesticides.

Reflecting these events, this year's State of the Environment Report to be released by the United Nations Environment Programme on the occasion of the World Environment Day (5 June) will focus upon the increasing dangers posed by pesticide resistance. The report makes quite frightening reading.

It points out that in 1965 , the Food and Agricultural Organisation listed 182 resistant strains of arthropod pests; in 1968, it listed 228 resistant species; and now its latest survey of 1977 lists 364 species.

There are now 223 agricultural pests resistant to nine of the major groups of pesticides. Many of these, says the UNEP report, "are major pests of major crops, such as cotton bollworm, the boll weevil, and the leafworm of cotton, the rice stem borer and the brown plant hopper, the Colorado beetle of potatoes, spider mites of fruit and glasshouse crops, and cutworms and weevils of cereals".

Up to 1970 , very few resistant plant pathogens had been observed. But with the introduction of new systemic fungicides, the resistance problem has crept in, and now more than 35 species of plant pathogens have been reported as resistant

Rodents, which destroy crops, are also becoming resistant to rodenticides. Latest FAO figures show that seven species of rodents, including two important and widespread species, Rattus rattus, and Rattus norvegicus, have developed resistant strains.

If there is any ray of hope it seems to be with weeds, which have not yet shown any sign of developing genetical resistance similar to that occurring amongst insects-even though herbicides now account for some $50 \%$ of all pesticides applied. For long-lived percnnial and vegetatively reproducing weeds, says the report, "the potential for development of genetical resistance seems low bearing in mind that experience with insects and other classes has demonstrated that in the field many generations must pass before such resistance reaches notice- able levels",

The most worrying situation is in the field of public health. WHO figures cited in the UNEP report show that there are now 121 resistant strains of insects important to public health campaigns compared to 102 in 1968 . In 1969, 15 species of anopheline mosquitoes were resistant to DDT and some 37 to dieldrin. In 1976, some 43 species were known to be resistant to dieldrin, 24 to DDT, five to organophosphates like malathion, and two to carbamates.

Resistance to insecticides is now found amongst anopheline mosquitoes in 62 countries out of 107 where there is malaria. In several parts of the world there has been a massive resurgence of the disease, with some countries showing a $30-40$ fold increase in the number of malaria cases since 1968-70.

Switching over from DDT to other insecticides like malathion has not proved easy. Most of the substitutes of DDT are both much more toxic and much more costly. India's malaria control programme alone consumes nearly $60 \%$ of the Indian government's health budget.

Amongst culicine mosquitoes, vectors of such diseases as yellow fever, filariasis and dengue, resistance has increased from 19 species in 1968 to 41 species in 1975. Other important vectors such as houseflies, black files and fleas are also becoming resistant. The house fly, says the UNEP report, seems to be the insect "with the greatest ability to develop resistance to insecticides over the widest geographical area". A total of 121 resistant strains of housefly were reported in 1975.

Research on pest control agents with novel modes of action like chemosteriliants, hormones and growth inhibitors, and biological agents like bacteria, viruses and fungi, has been hailed in the belief that pests would be less likely to develop resistance to them. The UNEP report however points out that this hope is ill-founded.

Resistance has already appeared to hormone-based pesticides where little resistance had been expected. "New compounds such as growth regulators and microbial pesticides have not been in use long enough or on a wide enough scale to show perceptible resistance, but here again as with chemosterilants, it has been possible in the laboratory to develop resistance by artificial selection."

The flour beetle, Tribolium, has not

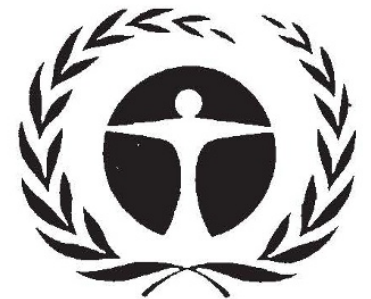

only developed multiple resistance to conventional pesticides but also acquired significant cross-resistance to the growth inhibitor, methoprene. It has also been shown that houseflies can become resistant both to the spores of Bacillus thuringiensis and to its toxin. B. thuringiensis is expected to become an important biological control agent.

What then is the solution to pest control? UNEP points out that the classical alternative approach of changing the pesticide when faced with the problem of resistance is a practical solution only in the short term. Alternative pesticides need to be developed but in the long term, the situation is such that it requires the development of alternative strategies.

Instead of total reliance on just one type of control agent like chemical pesticides. UNEP favours the concept of 'integrated pest control.' This would include elements of chemical pest control, environmental control of breeding habitats, genetic control techniques, biological control, behavioural control using sex pheromones and related compounds, and breeding of resistance in crops and animals.

Integrated pest control strategies will have to be specific to a pest in a specific environment. This will require considerable research. An FAO/ UNEP panel of experts on integrated pest control has over the last few years developed a Global Programme for Integrated Pest Control in relation to various priority crops like cotton, rice, sorghum, maize, millet, roots and tubers and grain legumes, and is now extending this to vegetable crops.

Studies conducted in South America using integrated pest control techniques on cotton-the highest consumer of insecticides in agricultureshows that the quantity of chemicals needed can be reduced by a third, thus cutting down costs and health risks and increasing productivity. But while this system of integrated pest management has been considerably developed, both in theory and in practice, as regards agricultural pest control, the UNEP report claims that extensive research programmes are still needed to apply it to public health vector control.

The switch from chemicals to the new packages of integrated practices is therefore likely to take a considerable time. 